\title{
Editorial
}

\section{Dynamic Systems and Related Algebra with Applications}

\author{
Chunrui Zhang, ${ }^{1}$ Baodong Zheng, ${ }^{2}$ and Liancheng Wang ${ }^{3}$ \\ ${ }^{1}$ Department of Mathematics, Northeast Forestry University, Harbin 150040, China \\ ${ }^{2}$ Department of Mathematics, Harbin Institute of Technology, Harbin 150001, China \\ ${ }^{3}$ Department of Mathematics and Statistics, Kennesaw State University, Kennesaw, GA 30144-5591, USA
}

Correspondence should be addressed to Chunrui Zhang; math@nefu.edu.cn

Received 12 August 2013; Accepted 12 August 2013

Copyright (C) 2013 Chunrui Zhang et al. This is an open access article distributed under the Creative Commons Attribution License, which permits unrestricted use, distribution, and reproduction in any medium, provided the original work is properly cited.

Dynamical systems have been widely used to describe the long-time behaviors in almost all areas of science and engineering. Using an algebraic approach to study dynamical systems has become more and more important in the dynamical systems theory and has drawn a great interest to many researchers recently.

Although dynamical systems theory and algebra seem to belong to two different areas of mathematics, they have a common generic structure. The study of the relationship between them has been active and very fruitful. The essence on this research is how to find different ways to associate algebra to dynamical systems in such a way that the algebra reflects the structure and the behavior of the solutions of the corresponding dynamical systems. As shown by the research, algebra can play a key role in the theory of dynamical systems. Topics in algebra such as similarity of matrices, eigenvalues, and (generalized) eigenspaces have been applied, recharacterized, and generalized in the dynamical systems theory. The most basic form of this interplay can be seen when a matrix $A$ gives rise to a dynamical system. Matrices define nonlinear systems on smooth manifolds. The behavior of systems is closely related to matrices and their properties [1-6].

In recent years this correspondence between dynamical systems and algebra has evolved rapidly, especially in the area of using the invariant theory of algebra in the classification of the dynamical systems [7-10]. In the late nineteenth century, in order to unify and extend the methods of solving ordinary differential equations, a Norwegian mathematician, Lie, inspired by Galois and Abel in their treatment of algebraic equations, introduced the concept of continuous Lie group.
The works of Lie were systematic and all around including integral factor, homogeneous equation, order reduction of linear equations, and Laplace transformation of Euler equation. Many important properties of solutions of differential equations can be represented and described by the corresponding matrix $A$. If $A$ is solvable, then the solution of the corresponding differential equation can be obtained through a linear differential equation. Automorphism keeps the equilibrium, periodic orbits, and the attraction region. Equilibrium of the differential equation can be obtained from the nilpotent element with index of $2\left(N^{2}=0\right)$ of $A$, and idempotents $\left(E^{2}=E\right)$ give ray solutions. The origin is never asymptotically stable, and the existence of nonzero idempotents implies that the origin is actually unstable.

The description of characteristics of dynamical systems by using algebra theory, especially matrix space and matrix algebra theory, can be regarded as a natural generalization of classical Galois theory. The dynamical systems in physical, biological, and other natural sciences usually have some special algebraic properties, and they are often very interesting. An important example is the equivariant bifurcation theory, which in a natural way can be regarded as the application of Lie group in symmetric system.

Symmetric dynamics is generated from a kind of special systems with symmetric properties in the state space. It is widely used in engineering, cell, crystal structure, and so on. It is highly nontrivial to determine the symmetries, the stabilities, and branching patterns for solutions of equations equivariant under a compact Lie group G. From a mathematical point of view, symmetry refers to the system as an invariant property in a group action [11-14]. The invariance means that 
the compact maps commute with the action of a compact Lie group, and the corresponding group is called the symmetric group. The action of a group known as the symmetry group is usually a closed subgroup of the general linear group such as the circle group $Z N$, which is the symmetry of a directed $n$-gon; cyclic group $S N$, which involves all permutations of $n$ objects; the dihedral group $D N$, which is the symmetry of a regular $n$-gon. In the traditional sense (Lie), there are two main topics in group theory analysis of dynamical systems: one is to find the corresponding symmetry group for a given differential equation; the other is to classify the given differential equation of the symmetric group. A general problem with basic meaning is to find invariants of matrix in the sense of topological equivalence. Often can the dynamic characteristics of the dynamic system be described by using the algebraic invariants. The equivariant Hopf bifurcation theorem states that bifurcating branches of periodic solutions with certain symmetries exist when the fixed-point subspace of that subgroup of symmetries is two dimensional. There is a group-theoretic restriction on the subgroup of symmetries in order for that subgroup to have a two-dimensional fixedpoint subspace in any representation. The theory of Hopf bifurcation in the presence of symmetry leads to algebraic questions concerning a Lie group. Equivariant bifurcation problems are dependent on the parameters of symmetric system.

When the parameters in a specific value change, some of its properties may vary. These variations are often dependent on the symmetry of the system. In the analysis of the algebraic theory of dynamical systems, people always first try to identify the corresponding dynamical system to allow a specific form of symmetry group that has been used widely. As H. Röhrl points out, a differential equation problem often is induced directly and is associated with the wonderful algebraic properties. Dynamical systems and related algebra problems have been proven to be significant in both mathematical theory and practical applications. The interplay between algebra and dynamical systems can solve some old problems, but, more importantly, it can create new opportunities in both areas [15-18].

Chunrui Zhang Baodong Zheng Liancheng Wang
[6] M. W. Hirsch, S. Smale, and R. L. Devaney, Differential Equations, Dynamical Systems, and an Introduction to Chaos, Elsevier, 2004.

[7] V. Ayala, F. Colonius, and W. Kliemann, "Dynamical characterization of the Lyapunov form of matrices," Linear Algebra and Its Applications, vol. 402, pp. 272-290, 2005.

[8] M. Golubitsky and D. Stewart, Singularities and Groups in Bifurcation Theory, Springer, New York, NY, USA, 1985.

[9] J. Wu, "Symmetric functional-differential equations and neural networks with memory," Transactions of the American Mathematical Society, vol. 350, no. 12, pp. 4799-4838, 1998.

[10] S. Guo and J. Wu, Bifurcation Theory of Functional Differential Equations, Springer, 2013.

[11] S. P. Banks, "The Lie algebra of a nonlinear dynamical system and its application to control," International Journal of Systems Science, vol. 32, no. 2, pp. 157-174, 2001.

[12] M. Golubitsky and I. Stewart, "An algebraic criterion for symmetric Hopf bifurcation," Proceedings of the Royal Society A, vol. 440, no. 1910, pp. 727-732, 1993.

[13] J.-E. Furter, A. M. Sitta, and I. Stewart, "Algebraic path formulation for equivariant bifurcation problems," Mathematical Proceedings of the Cambridge Philosophical Society, vol. 124, no. 2, pp. 275-304, 1998.

[14] M. Li and J. Muldowney, “On Bendixson's criterion," Journal of Differential Equations, vol. 106, pp. 27-39, 1993.

[15] N. Filipski and M. Golubitsky, "The abelian Hopf H mod K theorem," SIAM Journal on Applied Dynamical Systems, vol. 9, no. 2, pp. 283-291, 2010.

[16] S. Ruan and J. Wei, "On the zeros of transcendental functions with applications to stability of delay differential equations with two delays," Dynamics of Continuous, Discrete \& Impulsive Systems, vol. 10, no. 6, pp. 863-874, 2003.

[17] B. Zheng, L. Liang, and C. Zhang, "Extended Jury criterion," Science China Mathematics, vol. 53, no. 4, pp. 1133-1150, 2010.

[18] B. Zheng and L. Wang, "Spectral radius and infinity norm of matrices," Journal of Mathematical Analysis and Applications, vol. 346, no. 1, pp. 243-250, 2008.

\section{References}

[1] S. Walcher, Algebras and Differential Equations, Hadronic Press, Palm Harbor, Fla, USA, 1991.

[2] M. Hirsch, R. Devaney, and S. Smale, Differential Equations, Dynamical Systems, and Linear Algebra, Academic Press, 1974.

[3] C. Robinson, Dynamical Systems, CRC Press, Boca Raton, Fla, USA, 2nd edition, 1995.

[4] S. Wiggins, Introduction to Applied Nonlinear Dynamical Systems and Applications, Springer, 1996.

[5] J. Guckenheimer and P. Holmes, Nonlinear Oscillations, Dynamical Systems, and Bifurcations of Vector Fields, Springer, New York, NY, USA, 1983. 


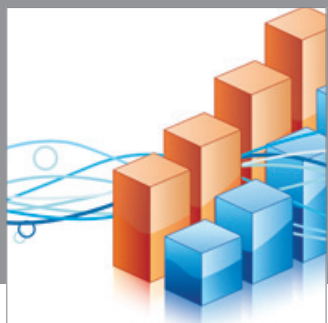

Advances in

Operations Research

mansans

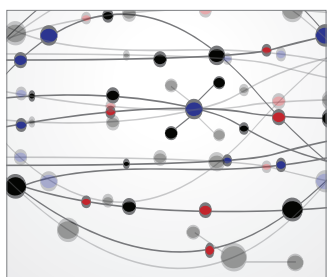

The Scientific World Journal
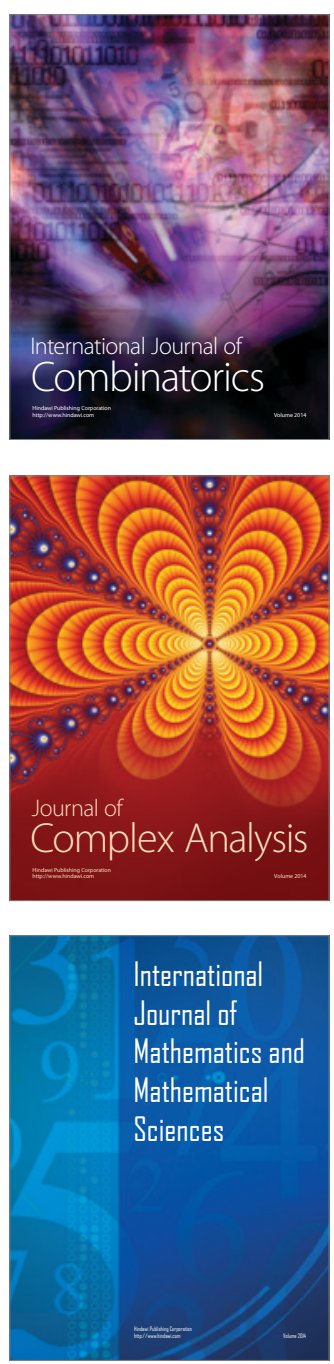
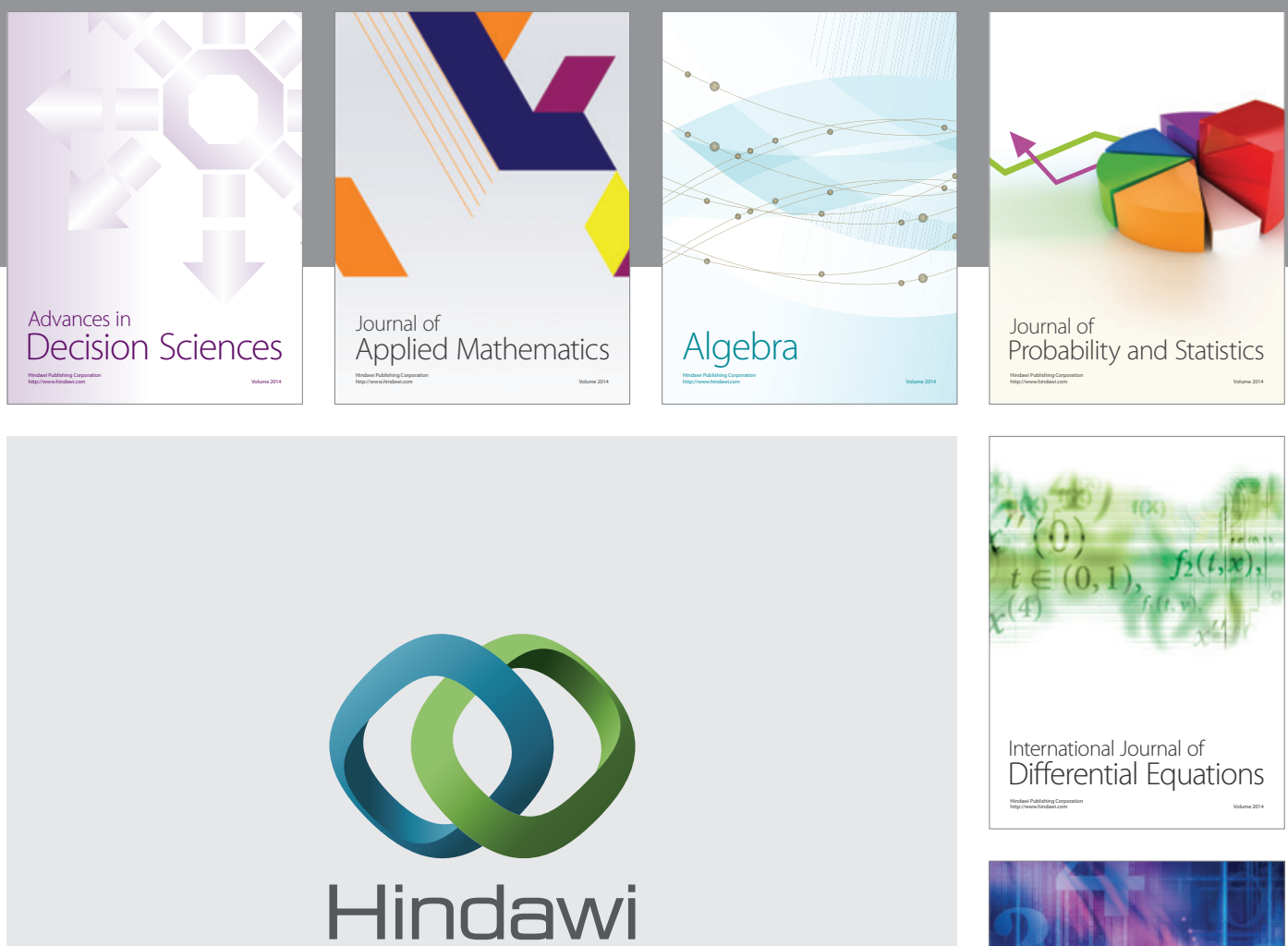

Submit your manuscripts at http://www.hindawi.com
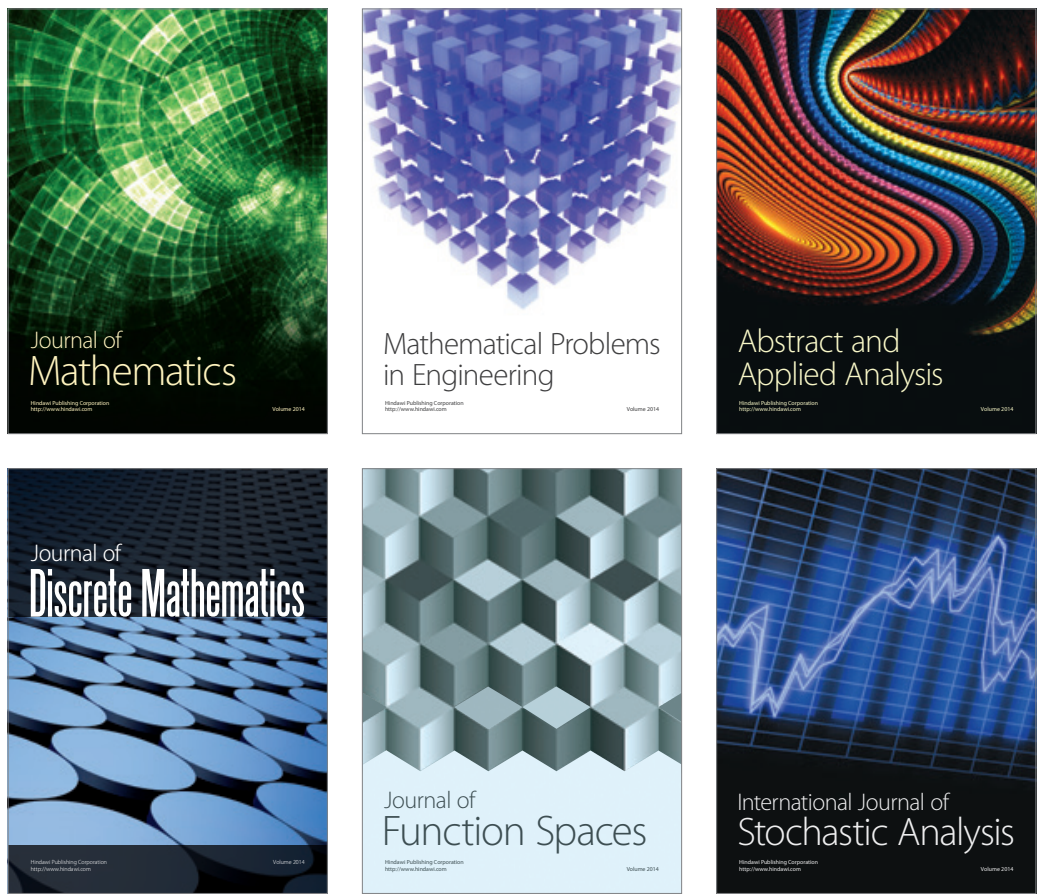

Journal of

Function Spaces

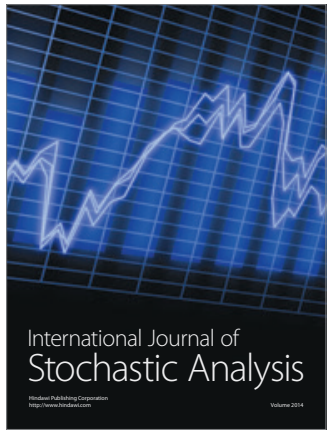

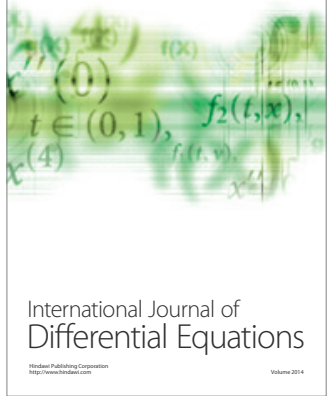
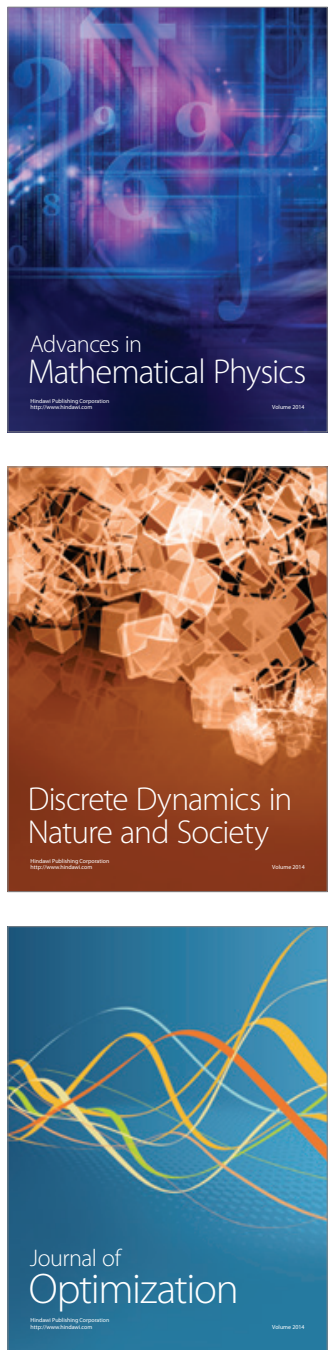